\title{
Quantitative Metallographic Study of the Solidification of Spheroidal Graphite Cast Iron*
}

\author{
By Toshiro Owadano**, Koji Yamada*** and Kiyoshi Torigoe****
}

\begin{abstract}
The eutectic solidification of $\mathrm{Fe}-\mathrm{Ni}-\mathrm{C}, \mathrm{Fe}-\mathrm{C}$ and $\mathrm{Fe}-\mathrm{Si}-\mathrm{C}$ spheroidal graphite cast irons were investigated quantitatively. Small specimens of the irons were quenched at various stages of solidification under controlled cooling, and their microstructures in vertical sections were examined.

The number of the growing graphite nodules or eutectic spheres in unit volume of the iron was found unchanged from the beginning of normal solidification. The growth rate constants of eutectic spheres obtained in a varied condition of undercooling were consistent with those calculated on the assumption that the growth was controlled by the carbon diffusion inward through the austenite envelope.

An approximate interrelation between the fraction and number of graphite nodules, the growth rate constant of eutectic spheres and the solidification time was obtained. From the equation and the saturated value of the growth rate constant, the critical conditions for the abnormal solidification to occur in the later stage were considered and verified experimentally. The relationship between undercooling and the number of graphite nodules in castings of the same solidification period was also successfully explained.
\end{abstract}

(Received September 12, 1977)

\section{Introduction}

Many investigators have studied the processes of eutectic solidification in spheroidal graphite irons by microscopic investigations of specimens quenched at various stages during solidification $^{(1)(2)}$. They found numbers of eutectic spheres growing during solidification in liquid, each of which was composed of a spheroidal graphite in the center and austenite envelope surrounding it. Although no satisfactory nucleation theory of eutectic sphere has been reported, it is generally accepted that the eutectic sphere grows by the transfer of carbon in liquid to the spheroidal graphite through the austenite envelope. Wetterfall

* This paper is abridged from papers published in Imono (J. of Japan Foundrymen's Society) 48 (1976), 23, 563 in Japanese.

** Kyushu Institute of Technology, Sensui-cho, Tobata-ku, Kitakyushu 804, Japan.

*** Graduate School, Kyushu Institute of Technology. Present address: Production Engineering Research Center, Osaka Factory, Komatsu Ltd., Ueno 3-chome, Hirakata 573, Japan.

**** Graduate School, Kyushu Institute of Technology, Sensui-cho, Tobata-ku, Kitakyushu 804, Japan.

Trans. JIM and his coworkers have proposed a diffusion controlled growth model of eutectic sphere in undercooled liquid ${ }^{(3)}$.

In this study we investigated the number and the growth rate of eutectic spheres in liquid by quantitative analyses of the specimens quenched during solidification in a varied condition of undercooling. The normal solidification process of spheroidal graphite iron was accounted for by the growth of eutectic spheres. Conditions for the abnormal solidification to occur in the later stage of solidification were also discussed.

\section{Mathematical Considerations}

\section{Growth of eutectic spheres in undercooled} liquid

According to Wetterfall and his coworkers ${ }^{(3)}$, a diffusion controlled growth model of a eutectic sphere in undercooled liquid is shown in Fig. 1. We will use the following notations hereafter:

$C_{\gamma}^{\boldsymbol{G} \gamma}$ : volumetric concentration of carbon in austenite at the graphite-austenite interface.

$C_{\gamma}^{\gamma L}$ : volumetric concentration of carbon in austenite at the austenite-liquid interface. 


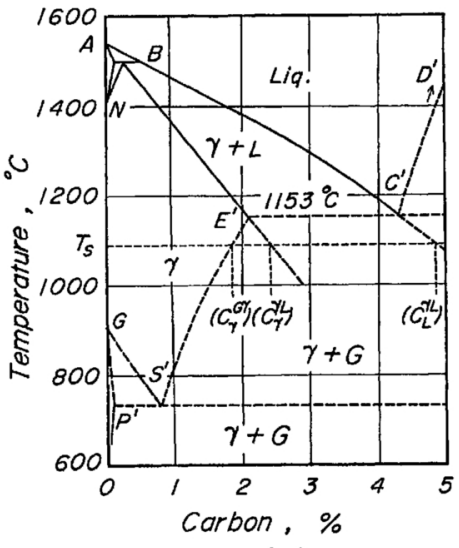

(a)

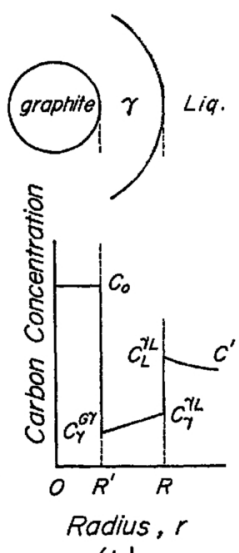

(b)
Fig. 1 Iron-graphite equilibrium diagram (a) and the growth model of eutectic sphere in liquid (b).

$C_{L}^{\gamma L}$ : volumetric concentration of carbon in liquid at the austenite-liquid interface.

$D_{C}^{\gamma}$ : diffusion coefficient of carbon in austenite.

$R$ : radius of eutectic sphere at time $t$.

$R^{\prime}$ : radius of graphite nodule at time $t$.

For the spherical diffusion in a quasisteady state shown in Fig. 1, Fick's first law yields

$$
4 \pi R^{2}\left(C_{L}^{\gamma L}-C_{\gamma}^{\gamma L}\right) d R=4 \pi \frac{C_{\gamma}^{\gamma L}-C_{\gamma}^{G \gamma}}{R-R^{\prime}} R R^{\prime} D_{C}^{\gamma} d t .
$$

When we put $\alpha=R / R^{\prime}$, eq. (1) results in

$$
(\alpha-1) R d R=K_{0} d t,
$$

where

$$
K_{0}=\frac{C_{\gamma}^{\gamma L}-C_{\gamma}^{G \gamma}}{C_{L}^{\gamma L}-C_{\gamma}^{\gamma L}} D_{C}^{\gamma}
$$

Although the value of $\alpha$ in the initial stage depends upon the nucleation mechanism of the eutectic sphere, it approaches rapidly 2.43 as the eutectic sphere grows, whatever its nucleation mechanism may be. (Appendix 1) Then, by integrating eq. (2), the steady state growth of the eutectic sphere during isothermal solidification may be written as

$$
R^{2}=2 K t+\text { constant, }
$$

where

$$
K=0.70 K_{0} .
$$

We will call $K$ the growth rate constant of the eutectic sphere. From eqs. (3) and (5), $K$ is expected to depend upon the solidification temperature.

\section{Process of bulk solidification}

The following assumptions are made:

(1) The number of eutectic spheres in unit volume of specimen $N_{V}$, which is equal to the number of graphite nodules in unit volume, is kept unchanged during solidification. The validity of this assumption will be proved later.

(2) All eutectic spheres are equi-sized.

The increment in solidified fraction $d S$ may be written as

$$
d S=(1-S) 4 \pi R^{2} N_{V} d R,
$$

where $S$ denotes the solidified fraction and the term $1-S$ is introduced in consideration of the mutual impingements of neighboring eutectic spheres. By integrating the above equation, we get

$$
S=1-\exp \left(-\frac{4}{3} \pi N_{V} R^{3}\right) .
$$

For conversion of $N_{A}$, the number of graphite nodules in unit area, to $N_{V}$ we will use

$$
N_{V}=\left(\frac{\pi}{6 V_{G}}\right)^{1 / 2}\left(1.2 N_{A}\right)^{3 / 2},
$$

where $V_{G}$ denotes the volume fraction of graphite nodules in the specimen $^{(4)}$. In a rough evaluation of the solidification progress we may neglect the constant term in eq. (4), which yields

$$
R^{2}=2 K t .
$$

Combining eqs. (6), (7) and (4'), we get

$$
S=1-\exp \left[-\left(1.6 V_{G}^{-1 / 3} N_{A} K t\right)^{3 / 2}\right]
$$

for isothermal solidification of spheroidal graphite iron.

\section{Experimental Procedures}

\section{Specimens}

Hyper-eutectic $\mathrm{Fe}-5 \% \mathrm{Ni}-\mathrm{C}$ and $\mathrm{Fe}-\mathrm{C}$ alloys were prepared by melting pure iron, electrolytic nickel and graphite powder and casting in sand molds. Each specimen weighing $10 \mathrm{~g}$ was cut from the prepared alloy and in- 


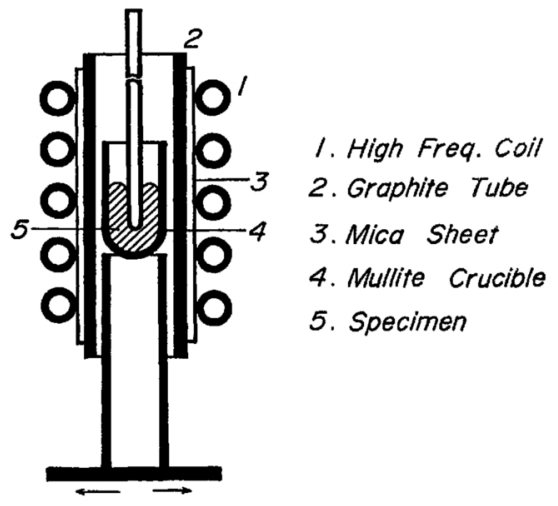

Fig. 2 Apparatus for experiment.

duction heated in a mullite crucible at $1400^{\circ} \mathrm{C}$ as shown in Fig. 2. A definite amount of $\mathrm{Fe}-75 \% \mathrm{Si}$ and electrolytic iron was added to adjust the chemical compositions in the experiments of $\mathrm{Fe}-\mathrm{Si}-\mathrm{C}$ alloy. $\mathrm{Ni}-15 \% \mathrm{Mg}$ spheroidizer was added on the surface of the molten specimen. After $1.5 \mathrm{~min}$ holding at $1400^{\circ} \mathrm{C}$, specimens were cooled by altering the induction current to a definite lower level. Some of the specimens were inoculated by $1 \%$ addition of $\mathrm{Fe}-75 \% \mathrm{Si}$ alloy at about $1250^{\circ} \mathrm{C}$ and stirred with a silica rod during cooling. Specimens as well as the crucibles were water quenched at various stages during solidification. Chemical compositions of the specimens and the added amounts of the Ni$\mathrm{Mg}$ alloy are tabulated in Table 1.

Table 1 Chemical analyses and spheroidizing.

\begin{tabular}{lcccc}
\hline Alloys & \multicolumn{3}{c}{ Chemical compositions, \% } & $\begin{array}{c}\text { Addition } \\
\text { of } \\
\text { Ni-Mg }\end{array}$ \\
\cline { 2 - 5 } & $\mathrm{C}$ & $\mathrm{Ni}$ & $\mathrm{Si}$ & $\begin{array}{c}\mathrm{Niloy,} \% \\
\text { alloy }\end{array}$ \\
\hline Fe-Ni-C & 4.20 & $7.0-7.3$ & - & 3 \\
Fe-C (A) & $4.24-4.29$ & $0.77-0.78$ & - & 1 \\
Fe-C (B) & 4.32 & 1.3 & - & 1.5 \\
Fe-Si-C & 3.27 & 2.6 & 3.30 & 3 \\
\hline \hline
\end{tabular}

\section{Analysis of microstructures}

The microstructures of the quenched specimens were examined in their vertical sections to confirm the homogeneity of the specimens. The number of graphite nodules in unit area $N_{A}$ was determined by counting the nodules in sectional area of $10 \mathrm{~mm}^{2}$ polished and etched lightly. Fractions of graphite nodules and eutectic spheres in area, which are supposed to be equal to their volume fractions ${ }^{(5)}$, were measured by point counting or line scanning.

The average radius of eutectic spheres $R$ in each specimen was computed by the following equation ${ }^{(6)}$ :

$$
R=\frac{3}{4} \bar{L}
$$

where $\bar{L}$ denoted the average length of intercept within the eutectic sphere in line scanning. Those intercepts which bridged the neighbouring spheres in impingement were excluded from the averaging.

\section{Experimental Results}

\section{Number and growth rate of eutectic spheres}

Representative cooling curves of the $\mathrm{Fe}$ $\mathrm{Ni}-\mathrm{C}$ alloys investigated are shown in Fig. 3. The fraction of graphite nodules $V_{G}$ in the $\mathrm{Fe}-\mathrm{Ni}-\mathrm{C}$ alloy quenched during solidification increases in proportion to the solidified fraction, satisfying the conditions of eutectic solidification as shown in Fig. 4. The number of graphite nodules in unit area $N_{A}$ increases during solidification, but the number of graphite nodules in unit volume $N_{V}$ calculated by eq. (7) is kept unchanged as shown in Fig. 5. As the austenite envelope is found around each of the graphite nodules, the solidification process is considered to be the growth of eutectic spheres at a definite number of sites determined in the early stage of solidification.

Squared radii of eutectic spheres $R^{2}$ measured

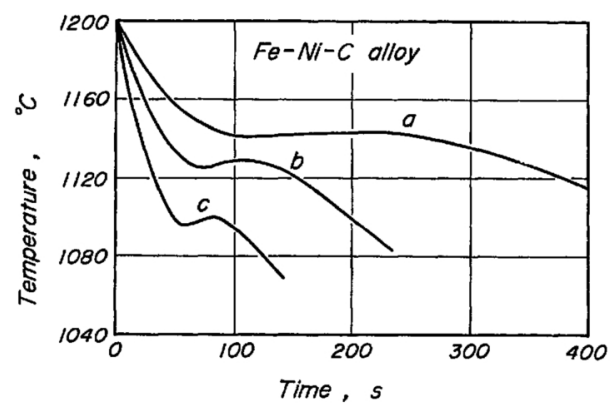

Fig. 3 Representative cooling curves of $\mathrm{Fe}-\mathrm{Ni}-\mathrm{C}$ specimens designated as cooling $\mathrm{a}, \mathrm{b}$ and $\mathrm{c}$. 


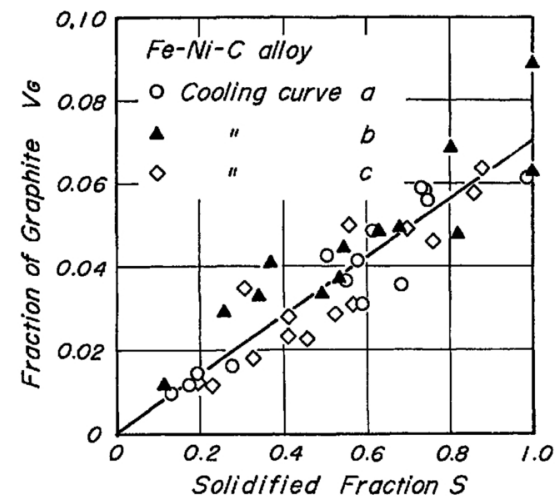

Fig. 4 Relation between fractions of graphite and solid prior to quenching.

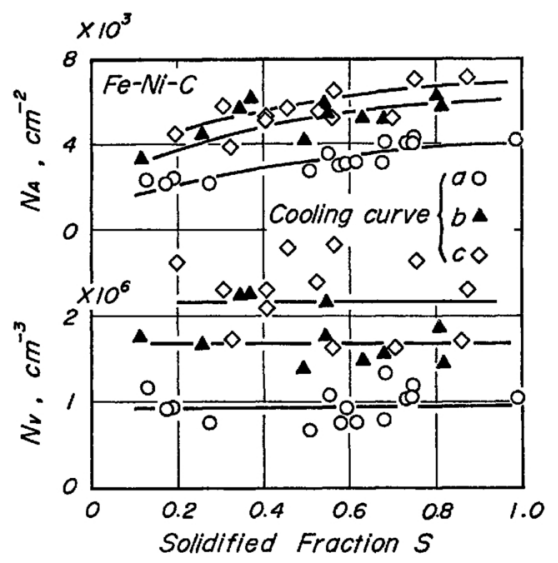

Fig. 5 The number of graphite nodules in unit area $N_{A}$ and the number in unit volume $N_{V}$ during solidification.

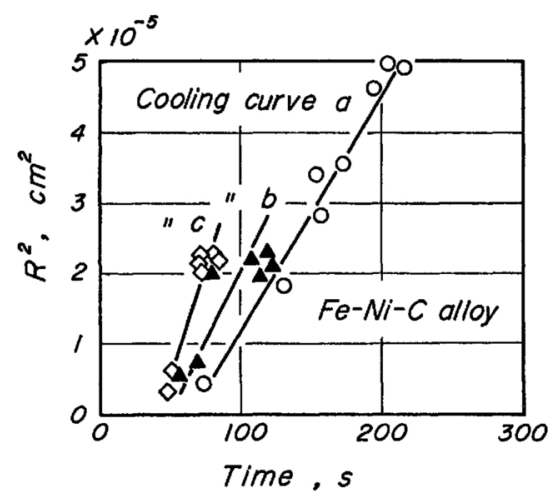

Fig. 6 Change in the average radii of eutectic spheres during solidification of $\mathrm{Fe}-\mathrm{Ni}-\mathrm{C}$ alloy specimens.

in the specimens quenched during solidification are shown in Fig. 6, in which the time on the abscisa is taken in the same way as in Fig. 3. Strictly speaking, the solidification processes investigated are not isothermal, so that the slopes of $R^{2}$ vs time plots in Fig. 6 may involve some errors in the evaluation of the growth rate constants $K$ by eq. (4). Nevertheless, the dependence of the average growth rate constants on the average solidification temperature may be revealed. In Fig. 7 the growth rate constants thus determined in $\mathrm{Fe}-\mathrm{Ni}-\mathrm{C}$ and $\mathrm{Fe}-\mathrm{C}$ alloys are plotted in relation to their average solidification temperature. The values of the growth rate constants calculated by eqs. (3) and (5) (Appendix 2) are also shown in Fig. 7. When the errors due to the anisothermal solidification are taken into account, the experimental and theoretical values of $K$ may be regarded as being in fairly good agreement. In other words, the growth of the eutectic spheres during solidification is probably controlled by the diffusion of carbon inward through the austenite envelope.

\section{Process of bulk solidification}

In order to quantitatively consider the solidified fraction as a function of time, it will be of use to determine the difference between the observed fractions in the actual solidification and the fractions calculated by eq. (8) for isothermal solidification. When we use eq. (8), the time of solidification $t$ must be known. In Fig. 8 are shown the conventional ways of determining the starting and ending points of solidification as well

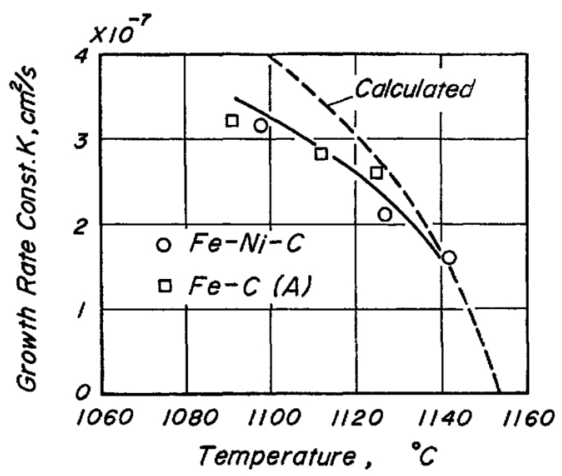

Fig. 7 Temperature dependence of the growth rate constant $K$. 


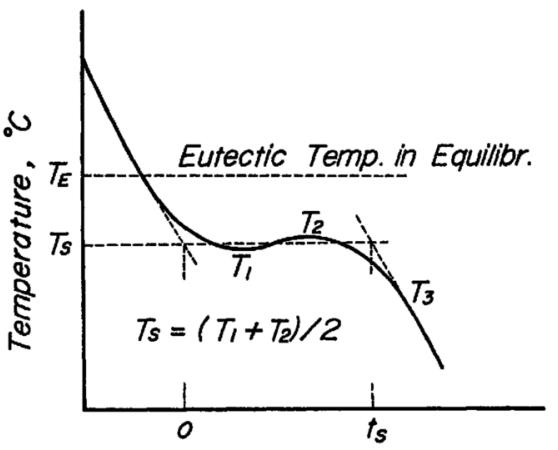

Time of Solidification $t$

Fig. 8 Conventional ways of determining the average solidification temperature and the start and end points of solidification in its cooling curve.

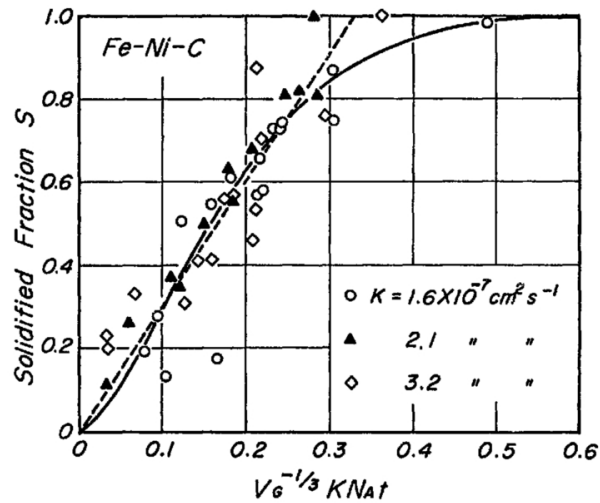

Fig. 9 Solidified fraction in relation to $V_{G}^{-1 / 3} N_{A} K t$. Solid line shows isothermal solidification calculated and broken line is supposed to correspond to the actual solidification.

as the solidification temperature $T_{s}$. The observed fractions of solidification are plotted in relation to the experimental values of $V_{G}^{-1 / 3} N_{A} K t$, together with a curve calculated by eq. (8) in Fig. 9. The calculated curve shows that the rate of solidification varies continuously during solidification. In the practical solidification, however, the gradual change in temperature and hence in $K$ seems to reduce partly the variation of the solidification rate. As a result, the observed fractions of solidification in Fig. 9 increase approximately in proportion to the time of solidification below $S=0.8$, beyond which the rate of solidification slows down. Then the solidification time $t_{s}$ may satisfy the following relationship as shown in Fig. 9:

$$
V_{G}^{-1 / 3} N_{A} K t_{S}=0.33 \text {. }
$$

$\mathrm{Fe}-\mathrm{C}(\mathrm{B})$ and $\mathrm{Fe}-\mathrm{Si}-\mathrm{C}$ alloys were used for the determination of $K$ values by eq. (10) for the different solidification temperatures induced by the change in cooling rate or the inoculation. Specimens were water quenched at about $1000^{\circ} \mathrm{C}$ after complete solidification. Figure 10 shows the average temperature of solidification $T_{S}$ and the number of graphite nodules in unit area $N_{A}$ in relation to the solidification time $t_{S}$. When $\mathrm{Fe}-\mathrm{C}(\mathrm{B})$ specimens were cooled at a relatively fast rate and $t_{\mathrm{S}}$ became less than about $40 \mathrm{~s}$, their cooling curves were always characterized by a sudden temperature rise in the later stage and ledeburite was found in their microstructures among the eutectic spheres as shown in Photo. 1. When $\mathrm{Fe}-\mathrm{Si}-\mathrm{C}$ specimens solidified in about 25 to $50 \mathrm{~s}$, there were no temperature rises in their cooling curves, but their microstructures showed fine graphite nodules dispersed along the boundaries of primary eutectic spheres as shown in Photo. 2. These fine graphite nodules were excluded from the counting of $N_{A}$, since they were considered to be formed in the later stage of solidification. When the specimens solidified within about $25 \mathrm{~s}$, however, ledeburite was formed in the same manner as in $\mathrm{Fe}-\mathrm{C}$ (B) specimens.

The measured fractions of graphite $V_{G}$

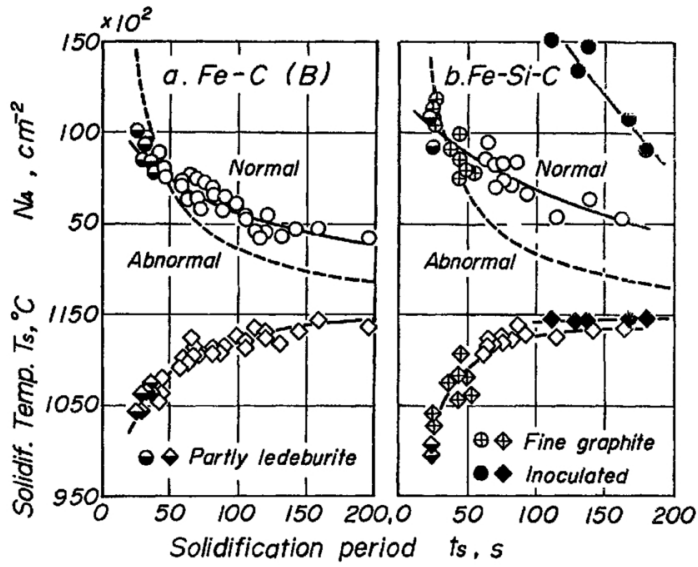

Fig. 10 Solidification temperature $T_{S}$ and the number of graphite nodules $N_{A}$ in relation to the solidification period $t_{\mathrm{s}}$. Broken lines show the calculated critical conditions for the transition from normal to abnormal solidification. 


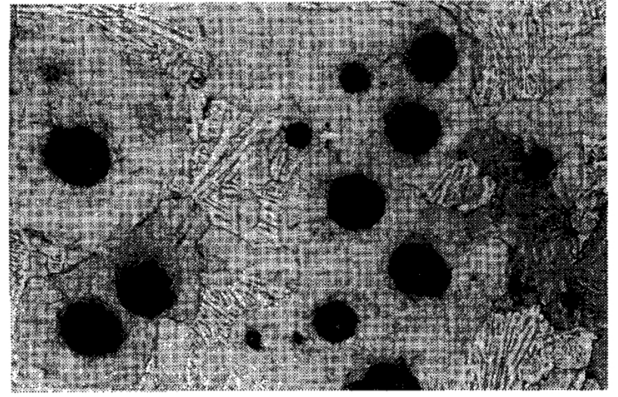

Photo. 1 Micrograph showing eutectic spheres enbeded in ledeburite. Fe-C specimen solidified in 30 s. $(\times 180 \times 0.7)$

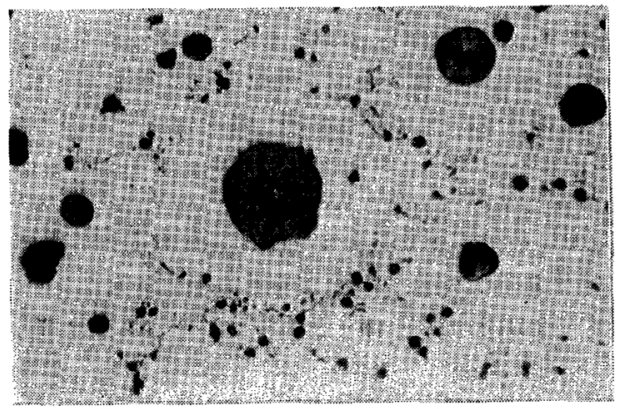

Photo. 2 Micrograph showing fine graphite nodules lined along the eutectic boundary. Fe-Si-C specimen solidified in $35 \mathrm{~s}$. $(\times 180 \times 0.7)$

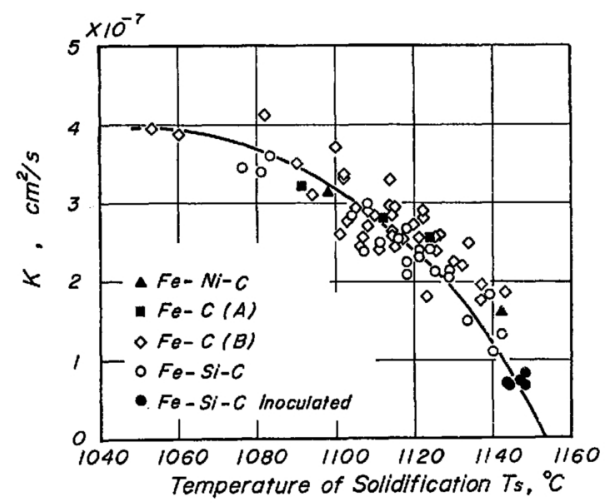

Fig. 11 The growth rate constant of eutectic sphere $K$ in $\mathrm{Fe}-\mathrm{C}, \mathrm{Fe}-\mathrm{Ni}-\mathrm{C}$ and $\mathrm{Fe}-\mathrm{Si}-\mathrm{C}$ alloys related to the average solidification temperature $T_{s}$.

were about 0.08 for $\mathrm{Fe}-\mathrm{C}$ (B) specimens and 0.06 for $\mathrm{Fe}-\mathrm{Si}-\mathrm{C}$ specimens respectively. The values of $K$ calculated by eq. (10) are shown in Fig. 11, together with those reproduced from Fig. 4. The temperature dependence of $K$ indicates almost no difference among
$\mathrm{Fe}-\mathrm{C}, \mathrm{Fe}-\mathrm{Ni}-\mathrm{C}$ and $\mathrm{Fe}-\mathrm{Si}-\mathrm{C}$ alloys, although some shift of the curve toward higher temperatures has been expected in $\mathrm{Fe}-\mathrm{Si}-\mathrm{C}$ alloy.

\section{Considerations}

\section{Ledeburite or fine graphite nodule forma- tion}

We have considered hitherto that the solidification of speroidal graphite iron is due to the diffusion controlled growth of eutectic spheres at a definite number of sites. In $\mathrm{Fe}-\mathrm{C}$ (B) specimens undercooled below $1050^{\circ} \mathrm{C}$, however, the ledeburite is formed as shown in Fig. 10(a). Since $K$ is approximately $4 \times 10^{-7}$ $\mathrm{cm}^{2} / \mathrm{s}$ at this temperature, the critical conditions for a specimen to be undercooled to this temperature may be written from eq. (10) as

$$
N_{A} t_{S}=3.55 \times 10^{5} \mathrm{~s} / \mathrm{cm}^{2} .
$$

Similarly, the critical conditions for the $\mathrm{Fe}$ $\mathrm{Si}-\mathrm{C}$ specimens with $V_{G}=0.06$ to be undercooled below $1000^{\circ} \mathrm{C}$ may be written as

$$
N_{A} t_{S}=3.23 \times 10^{5} \mathrm{~s} / \mathrm{cm}^{2} .
$$

The conditions thus calculated are shown by the broken lines in Fig. 10. The normal and abnormal (partly ledeburite) solidifications are likely to be clearly divided by the calculated curves.

The fine graphite nodules are formed in the $\mathrm{Fe}-\mathrm{Si}-\mathrm{C}$ specimens solidified near the critical condition as shown in Fig. 10(b). Therefore, the increased silicon content in this alloy seems to result in an increase of graphite nodule number in the later stage as well as in the early stage of solidification.

Figure 10 shows, moreover, that the increased number of graphite nodules or eutectic spheres by inoculation can change the abnormal structure for the normal structure in the casting of a definite solidification time, although the exact mechanism of inoculation is not known.

\section{Chart for computation of $N_{A}$}

Equation (10) and the values of $K$ shown in Fig. 11 may serve to evaluate the number of graphite nodules in unit area $N_{A}$, which 


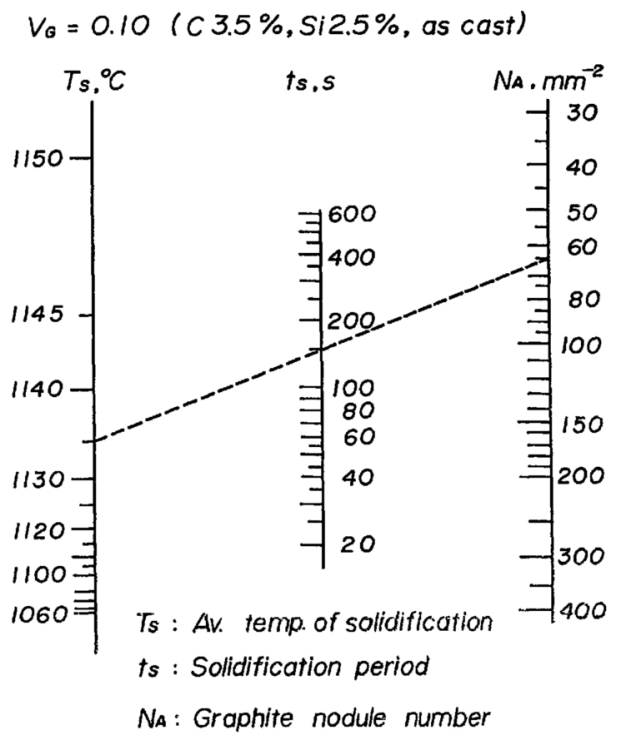

Fig. 12 A chart showing the relationship among the solidification temperature $T_{S}$, solidification period $t_{S}$ and the number of graphite nodules in unit area $N_{A}$ in $\mathrm{Fe}-\mathrm{Si}-\mathrm{C}$ alloy castings with $V_{G}=0.10 . N_{A}$ can be determined by this chart.

has been reported to be a matrix controlling factor $^{(7)}$. A chart for computation of $N_{A}$ in $\mathrm{Fe}-\mathrm{Si}-\mathrm{C}$ alloy castings with $V_{G}=0.10$ is shown in Fig. 12. When its average temperature of solidification $T_{S}$ and the solidification time $t_{S}$ are known from its cooling curve, the approximate values of $N_{A}$ can be determined by the chart. The broken line in Fig. 12 shows that when $T_{S}=1135^{\circ} \mathrm{C}$ and $t_{S}=150 \mathrm{~s}$, for instance, the expected $N_{A}$ becomes $65 \mathrm{~mm}^{-2}$. We can recognize that the more the number of graphite nodules, the higher becomes its solidification temperature, if the solidification time is constant. This may be the reason why the inoculated castings have a higher temperature of solidification than the non-inoculated ones.

\section{Conclusion}

The microstructures in quenched specimens during and after solidification of spheroidal graphite irons were analyzed quantitatively. The results of this study are summarized as follows:

(1) The number of graphite nodules or eutectic spheres in unit volume is kept unchanged during solidification, and the bulk solidification proceeds normally owing to the growth of these eutectic spheres.

(2) The growth rate constant of eutectic spheres in liquid increases and approaches a saturation value as the solidification temperature is lowered. $\mathrm{Fe}-\mathrm{Ni}-\mathrm{C}, \mathrm{Fe}-\mathrm{C}$ and $\mathrm{Fe}-$ $\mathrm{Si}-\mathrm{C}$ alloys investigated make almost no difference in the temperature dependence of the growth rate constant. The growth rate constants obtained from the experiments are consistent with those calculated on the assumption that the growth is diffusiondependent.

(3) The interrelation among the fraction of graphite $V_{G}$, the number of graphite nodules in unit area $N_{A}$, the growth rate constant $K$ and the solidification time $t_{S}$ in the normal solidification can be approximately expressed as

$$
V_{G}^{-1 / 3} N_{A} K t_{S}=0.33 .
$$

The critical conditions for the abnormal solidification to occur are derived from the above interrelation and the values of $K$. Moreover, a chart for the evaluation of the number of graphite nodules from the cooling curve of a casting is obtained.

\section{Acknowledgment}

The authors wish to manifest their thanks to Messrs. I. Kanzaki, O. Takasaki, S. Nanba, Y. Kanagawa and Y. Inoue, T. Nishizawa, of the Kyushu Institute of Technology, for their assistance in the experiments of this study.

\section{REFERENCES}

(1) H. Morrogh: The Solidification of Metals, The Iron and Steel Inst. London, (1968), p. 238.

(2) Casting Subcommittee, The Japan Inst. Metals: Theory and Practice of Spheroidal Graphite Cast Irons, Maruzen, (1966), p. 169, 187.

(3) S. E. Wetterfall, H. Frederiksson and M. Hillert: J. Iron Steel Inst., 210 (1972), p. 323.

(4) T. Owadano: Imono (J. of Japan Foundrymen's Society), 45 (1973), p. 193.

(5) R. F. DeHoff and F. N. Rhines: Quantitative Microscopy, McGraw-Hill Book Co., (1968), p. 45 .

(6) R. L. Fullman: Trans. AIME, 197 (1953), p. 447. 
(7) T. Owadano, T. Nishimura, H. Miyata and $Y$. Kubo: Trans. JIM, 16 (1975), p. 663.

(8) C. Wells, W. Bats and R. F. Mehl: Trans. AIME, 188 (1950), p. 553.

\section{Appendix 1}

If a spheroidal graphite nodule nucleates and grows in liquid until its radius becomes $R_{0}$ when the austenite envelope is formed, the balance in carbon is expressed by

$$
\begin{aligned}
\left(R^{3}-R_{0}^{3}\right) & \left(C^{\prime}-\frac{C_{\gamma}^{G \gamma}+C_{\gamma}^{\gamma L}}{2}\right) \\
& =\left(R^{\prime 3}-R_{0}^{3}\right)\left(C_{0}-\frac{C_{\gamma}^{G \gamma}+C_{\gamma}^{\gamma L}}{2}\right),
\end{aligned}
$$

where $C_{0}$ and $C^{\prime}$ denote the volumetric concentration of carbon in graphite and eutectic iron respectively. By putting

$$
\begin{aligned}
\beta & =R / R_{0}, \\
A & =\left(C_{0}-\frac{C_{\gamma}^{G \gamma}+C_{\gamma}^{\gamma L}}{2}\right) \\
& /\left(C^{\prime}-\frac{C_{\gamma}^{G \gamma}+C_{\gamma}^{\gamma L}}{2}\right)=14.3,
\end{aligned}
$$

we get

$$
\alpha=\left(\frac{A}{\beta^{3}-1+A}\right)^{1 / 3} .
$$

If a supersaturated austenite sphere with radius $R_{0}$ solidifies when a small spheroidal graphite nodule precipitates in its center, the balance in carbon is expressed by

$$
\begin{aligned}
\left(R^{3}-R_{0}^{3}\right)\left(C^{\prime}-\frac{C_{\gamma}^{G \gamma}+C_{\gamma}^{\gamma L}}{2}\right) \\
=R^{\prime 3}\left(C_{0}-\frac{C_{\gamma}^{G \gamma}+C_{\gamma}^{\gamma L}}{2}\right)
\end{aligned}
$$

Then we get

$$
\alpha=\left(\frac{A}{\beta^{3}-1}\right)^{1 / 3} .
$$

If a spheroidal graphite and austenite envelope nucleates in cooperation as a eutectic

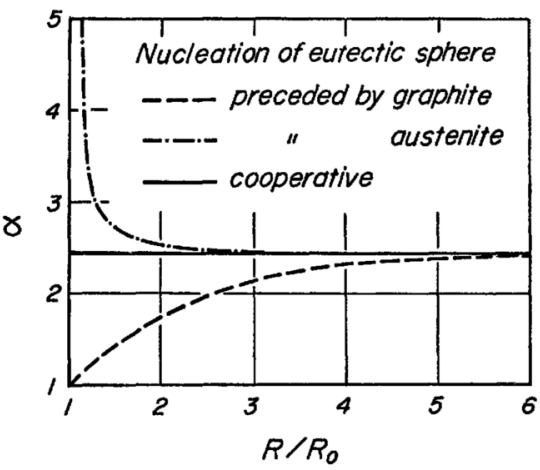

Fig. 13 Change in $\alpha$ with $\beta$ in three different nucleation models of eutectic spheres.

sphere in liquid,

$$
\alpha=A^{1 / 3} .
$$

The calculated values of $\alpha$ in these three cases are shown in relation to $\beta$ in Fig. 13. The values of $\alpha$ can be determined experimentally by

$$
\alpha=R / R^{\prime}=\left(S / V_{G}\right)^{1 / 3} .
$$

The straight line in Fig. 5 corresponds to $\alpha=A^{1 / 3}=2.43$, which approximates the experimental data. So that, we should consider that the steady state growth of eutectic spheres is achieved since the early stage of solidification.

\section{Appendix 2}

The growth rate constants of eutectic spheres in liquid were calculated from eqs. (3) and (5) by putting

$$
\begin{aligned}
& C_{\gamma}^{\gamma L}-C_{\gamma}^{\gamma G}=7.9 \times 10^{-4} \Delta T \mathrm{~g} / \mathrm{cm}^{3}, \\
& C_{L}^{\gamma L}-C_{\gamma}^{\gamma L}=0.144 \mathrm{~g} / \mathrm{cm}^{3}, \\
& D_{C}^{\gamma}=0.07 \exp (-28500 / R T) \mathrm{cm}^{2} / \mathrm{s}^{(8)},
\end{aligned}
$$

where $\Delta T$ : undercooling below $1153^{\circ} \mathrm{C}$

$$
\begin{aligned}
R: & \text { gas constant } \\
T: & \text { absolute temperature of solidi- } \\
& \text { fication. }
\end{aligned}
$$

